\title{
Retrospective Analysis of Drug Sensitivity of Neisseria gonorrhoeae in Teaching Hospitals of South China
}

\author{
Bingming Zhu ${ }^{\mathrm{l}, *}$ \\ Yanqing $\mathrm{Hu}^{2,3}$ \\ Xianyi Zhou ${ }^{2,3}$ \\ Kangxing Liu $^{2,3, *}$ \\ Wangrong Wen' \\ Yongxuan $\mathrm{Hu}$ (iD) 2,3 \\ 'Department of Clinical Laboratory, The \\ First Affiliated Hospital of Jinan \\ University, Guangzhou, Guangdong, \\ People's Republic of China; ${ }^{2}$ Department \\ of Dermatology and Venereology, The \\ 3rd Affiliated Hospital of Southern \\ Medical University, Guangzhou, People's \\ Republic of China; ${ }^{3}$ Guangdong Provincial \\ Key Laboratory of Bone and Joint \\ Degeneration Diseases, Guangzhou, \\ People's Republic of China \\ *These authors contributed equally to \\ this work
}

\begin{abstract}
The aim of this study was retrospective analysis of drug sensitivity of Neisseria gonorrhoeae in two teaching hospitals of South China. A total of 304 Neisseria gonorrhoeae isolates obtained from patients in South China from 2016 to 2020 were evaluated. The MICs of penicillin, cefuroxime, ceftriaxone (CRO), cefepime, ciprofloxacin, ceftazidime and azithromycin (AZM) against the isolates were determined by the agar dilution method. Then, Neisseria gonorrhoeae isolates were categorized into sensitive, moderately sensitive and resistant according to MICs. Also, $\beta$-lactamases were detected by enzyme linked immunosorbent assay (ELISA). Ureaplasma urealyticum and Mycoplasma hominis were determined by culture in liquid medium, and Chlamydia was detected by rapid antigen test. The result showed there was $50.99 \%, 20.72 \%, 9.87 \%, 14.47 \%, 86.84 \%, 7.57 \%, 6.91 \%, 11.18 \%$ resistance to penicillin, cefuroxime, ceftriaxone, cefepime, ciprofloxacin, ceftazidime and azithromycin, respectively. Also, $\beta$-lactamase positivity was $53.29 \%$ and Chlamydia antigen positivity was $20.07 \%$. Ureaplasma urealyticum and Mycoplasma hominis positivity was $11.84 \%$ and $6.25 \%$, respectively. From 2016 to 2020, the resistant rate of ceftriaxone and azithromycin gradually increased. In conclusion, Southern China is among the area reporting gonococci with high-level resistance to AZM and CRO, so N. gonorrhoeae culture and drug sensitivity test will be vital for monitoring trends in antimicrobial resistance.
\end{abstract}

Keywords: drug sensitivity, Neisseria gonorrhoeae, retrospective analysis

\section{Introduction}

Neisseria gonorrhoeae infection, one of the most common sexually transmitted disease, is a global problem. ${ }^{1}$ In mainland china, the incidence of $N$. gonorrhoeae infection has increased continuously, with the incidence rate among the top five of national notifiable infectious diseases. ${ }^{2}$ A wide spectrum of clinical manifestations and complications of $N$. gonorrhoeae infection has been observed, and the vaccine against $N$. gonorrhoeae is not available at present. Therefore, the infection control predominantly relies on timely diagnosis and appropriate antibiotic therapy. ${ }^{1-4}$ Over time, Neisseria gonorrhoeae has developed resistance to a broad range of antimicrobial agents. Some sensitive antibiotics, such as ceftriaxone, have been reported to have become ineffective in the recent years. ${ }^{5,6}$ The rapid development of $N$. gonorrhoeae resistance to antimicrobial agents worldwide will threaten the management of $N$. gonorrhoeae infection. ${ }^{1}$

This study is a retrospective analysis of drug sensitivity of Neisseria gonorrhoeae in the two teaching hospitals of South China from 2016 to 2020. 


\section{Materials and Methods}

Retrospective analysis of drug sensitivity of Neisseria gonorrhoeae in two teaching hospitals of Guangzhou, South China from 2016 to 2020 was performed.

The demographic and clinical information of patients such as gender, age, and symptoms was collected using electronic case system. Each isolate was cultured and verified. $^{7}$ MIC for penicillin, cefuroxime, ceftriaxone (CRO), cefepime, ciprofloxacin, ceftazidime and azithromycin (AZM) were determined by the agar dilution method. Antimicrobial susceptibility was interpreted according to criteria defined by WHO. ${ }^{8,9}$

The $\beta$-lactamase was detected by enzyme linked immunosorbent assay (ELISA). ${ }^{10}$ Ureaplasma urealyticum and Mycoplasma hominis was determined by culture in liquid medium, and Chlamydia was detected by rapid antigen test. ${ }^{10}$ All the results were collected using electronic case system and clinical examination electronic system.

Statistical significance was assessed using SPSS 18.0. Chi-square tests were used for statistical analyses. A $P<$ 0.05 was considered to be significant.

\section{Results}

Three hundred and four patients with Neisseria gonorrhoeae infection were included in this study. The study included 282 male and 22 females. The age range was from 16 to 75 years, with the average age $30.30 \pm 9.11$ years. From 2016 to 2020 , resistance rate to ceftriaxone was $6.38 \%, 8.06 \%, 8.86 \%, 14.29 \%$ and $11.67 \%$, respectively, the average resistance was $9.87 \%$. Resistance to azithromycin was $6.38 \%, 9.68 \%, 10.13 \%, 12.50 \%$ and $16.67 \%$, respectively, in 2016 to 2020 , and the average resistance was $11.18 \%$ (Table 1). The differences in the resistance rate between 2016 and 2020 were statistically significant for ceftriaxone and azithromycin $(P<0.05)$.

From 2016 to 2020, $\beta$-lactamase positive of Neisseria gonorrhoeae was rising every year $(P<0.05)$. Neisseria gonorrhoeae infection combined with Chlamydia infection and Ureaplasma urealyticum infection was also increased gradually $(P<0.05)$ (Table 2$)$.

\section{Discussion}

The vaccine against N.gonorrhoeae is not available at present; a wide spectrum of clinical manifestations and complications of $N$. gonorrhoeae infection have increased gradually. Therefore, N.gonorrhoeae infection control

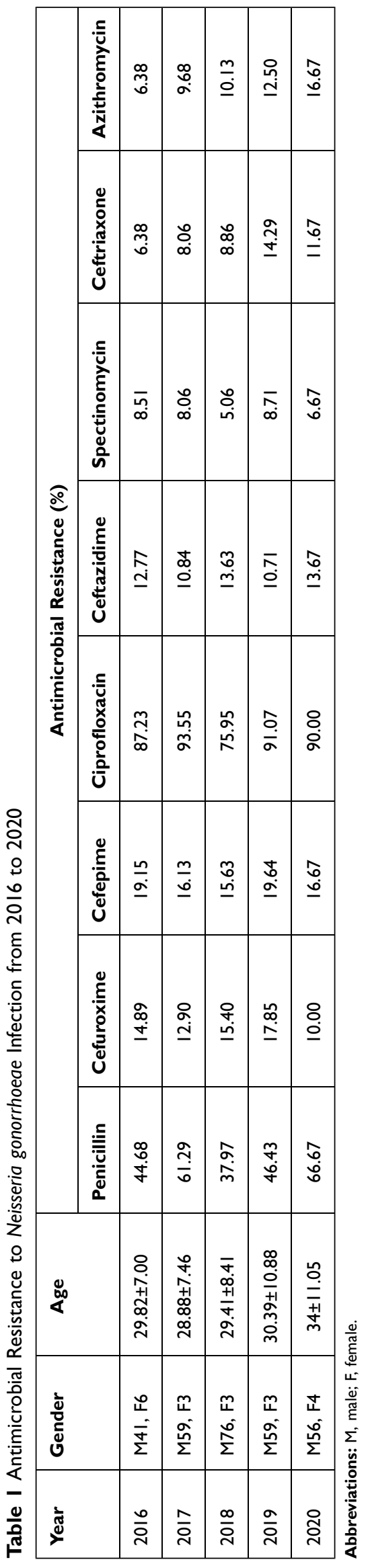




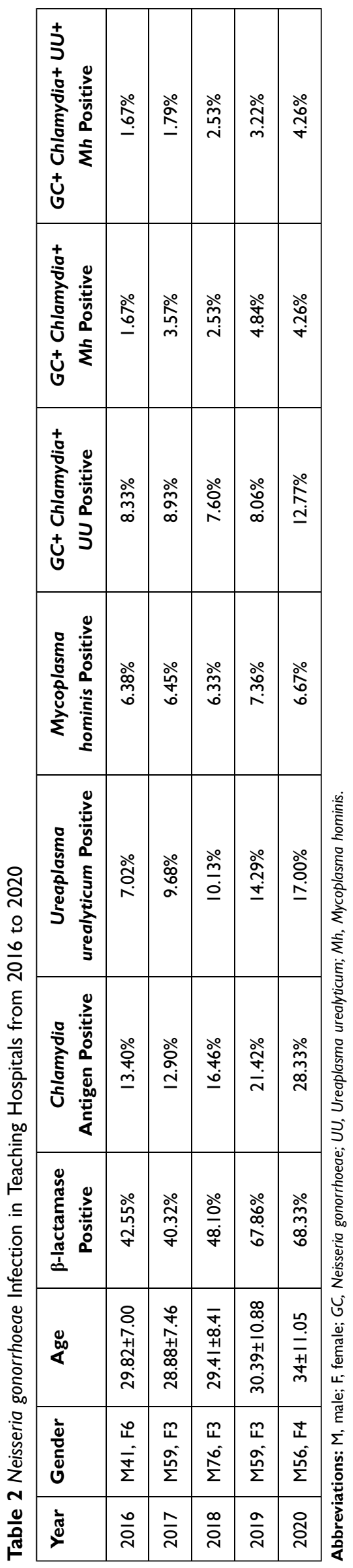

predominantly relies on accurate diagnostics and timely antibiotic therapy following antibiotic sensitivity testing. ${ }^{1-4}$

In the recent years, the management of N.gonorrhoeae infection has been difficult because of the rapid development of resistance to a broad range of antimicrobial agents. ${ }^{1}$

In order to limit the development of antimicrobial resistance and ensure effective treatment, the therapy with ceftriaxone 250 or $500 \mathrm{mg}$ administered intramuscularly plus azithromycin 1 or $2 \mathrm{~g}$ orally has been widely accepted as the first choice for the treatment of $N$. gonorrhoeae infection. ${ }^{11}$ As the emergence of treatment failure with cephalosporins have been documented in several countries in the 2000 s and the warning susceptibility of $N$. gonorrhoeae, some scholars recommend the use of $1000 \mathrm{mg}$ ceftriaxone intramuscularly. ${ }^{3}$ In this retrospective study, $N$. gonorrhoeae resistance rate to ceftriaxone was $6.38 \%, 8.06 \%, 8.86 \%, 14.29 \%$ and $11.67 \%$, respectively, from 2016 to 2020 . The average resistance was $9.87 \%$. This is consistent with previous studies. ${ }^{5-7}$

Situated in Southern China, Guangzhou is the important node city of "the Silk Road" and "The Belt and Road", which has a large transient population of foreign visitors and business partners. The import and dissemination of antimicrobial resistance is a constant public health threat in Guangzhou. Ceftriaxone-resistant multidrugresistant $N$. gonorrhoeae undermines the effectiveness of currently recommended first-line dual therapy. It is imperative to enhanced surveillance of antimicrobial susceptibility, in order to detect and monitor the resistance trends of Neisseria gonorrhoeae, so as to ensure the effectiveness of its treatment. ${ }^{1,3}$

In this study, resistance rate from 2016 to 2020 has increased significantly with regard to ceftriaxone and azithromycin. Also, Neisseria gonorrhoeae infection combined with Chlamydia infection and Ureaplasma urealyticum infection has also increased gradually. Pathogenic examination, especially $N$. gonorrhoeae culture and drug sensitivity test, is more important for the selection of sensitive antibiotics. A limitation of this study is that a small number of cases were assessed.

\section{Ethics Statement}

The study was approved by the Ethics Committee of the First Affiliated Hospital of Jinan University and the Third Affiliated Hospital of Southern Medical University. All subjects were adults and provided written informed consent. 
This study was conducted in accordance with the Declaration of Helsinki.

\section{Acknowledgments}

This work was supported by grants from the Scientific Research Project of Traditional Chinese Medicine Bureau of Guangdong Provincial (grant no-20212147).

\section{Author Contributions}

All authors made substantial contributions to conception and design, acquisition of data, or analysis and interpretation of data; took part in drafting the article or revising it critically for important intellectual content; agreed to submit to the current journal; gave final approval of the version to be published; and agree to be accountable for all aspects of the work.

\section{Disclosure}

The authors have no conflicts of interest to declare.

\section{References}

1. Unemo M, Bradshaw CS, Hocking JS, et al. Sexually transmitted infections: challenges ahead. Lancet Infect Dis. 2017;17(8):e235e279.

2. Wan C, Li Y, Le W-J, et al. Increasing resistance to azithromycin in Neisseria gonorrhoeae in Eastern Chinese cities: resistance mechanisms and genetic diversity among isolates from Nanjing. Antimicrob Agents Chemother. 2018;62(5):e2499-17. doi:10.1128/AAC.02499-17
3. Liu Y-H, Wang Y-H, Liao C-H, Hsueh P-R. Emergence and spread of Neisseria gonorrhoeae strains with high-level resistance to azithromycin in Taiwan from 2001 to 2018. Antimicrob Agents Chemother. 2019;63(9):e00773-19. doi:10.1128/AAC.00773-19

4. Yao H-Y, Wang C-H. Preseptal cellulitis caused by Neisseria gonorrhoeae: a rare disease need to be vigilant. J Microbiol Immunol Infect. 2017;50(3):397-398. doi:10.1016/j.jmii.2015.05.024

5. Ko KKK, Chio MTW, Goh SS, et al. First case of ceftriaxone-resistant multidrug-resistant Neisseria gonorrhoeae in Singapore. Antimicrob Agents Chemother. 2019;63(5):e02624-18. doi:10.1128/AAC.02624-18

6. Chen S-C, Yin Y-P, Chen X-S. Cephalosporin-resistant Neisseria gonorrhoeae clone, China. Emerg Infect Dis. 2018;24(4):804-806. doi:10.3201/eid2404.171817

7. Liang J-Y, Cao W-L, Li X-D, et al. Azithromycin-resistant Neisseria gonorrhoeae isolates in Guangzhou, China (2009-2013): coevolution with decreased susceptibilities to ceftriaxone and genetic characteristics. BMC Infect Dis. 2016;16:152. doi:10.1186/s12879-016-1469-3

8. World Health Organization. Global action plan to control the spread and impact of antimicrobial resistance in Neisseria gonorrhoeae; 2012. Available from: http://www.who.int/reproductivehealth/publica tions/rtis/9789241503501/en/. Accessed October 19, 2015.

9. Lusti-Narasimhan M, Pessoa-Silva CL, Temmerman M. Moving forward in tackling antimicrobial resistance: WHO actions. Sex Transm Infect. 2013;89(Supp14):iv57-iv59. doi:10.1136/sextrans-2012050910

10. Tsevat DG, Wiesenfeld HC, Parks C, Peipert JF. Sexually transmitted diseases and infertility. Am J Obstet Gynecol. 2017;216(1):1-9. doi:10.1016/j.ajog.2016.08.008

11. Unemo M, Shafer WM. Antimicrobial resistance in Neisseria gonorrhoeae in the 21st century: past, evolution, and future. Clin Microbiol Rev. 2014;27(3):587-613.
Infection and Drug Resistance

\section{Publish your work in this journal}

Infection and Drug Resistance is an international, peer-reviewed openaccess journal that focuses on the optimal treatment of infection (bacterial, fungal and viral) and the development and institution of preventive strategies to minimize the development and spread of resistance. The journal is specifically concerned with the epidemiology of

\section{Dovepress}

antibiotic resistance and the mechanisms of resistance development and diffusion in both hospitals and the community. The manuscript management system is completely online and includes a very quick and fair peerreview system, which is all easy to use. Visit http://www.dovepress.com/ testimonials.php to read real quotes from published authors. 